\title{
Substance Index for Volume 70
}

Published online: 27 February 2018

(c) Japan Antibiotics Research Association 2018

$\mathrm{A} 201 \mathrm{~A}$

(R)-7-Acetyl-3,6-dihydroxy-8-propyl-3,4-dihydronaphthalen-1 $(2 H)$-one

Achromosin

Acremopeptin

Allantopyrone A

Allocyclinone A, B, C, D

Amycolatopsin A, B, C

Antarlide F, G, H

Aquayamycin B, dehydroxy

Arabilin

Arugosin K

AS2488053

AS2488059

AS2524371

AS2529132

ASP2397

Aspergone

Aurachin SS

Avermectin $\mathrm{B}_{1 \mathrm{a}}, \mathrm{B}_{1 \mathrm{~b}}$

Azithromycin

(E)-3-(2-(1H-Benzo[d] imidazol-1-yl)-1-(((4chlorobenzyl)oxy)imino)ethyl)-2 $H$-chromen-2-one

(2S,4R)-2-Benzyl 1-tert-butyl 4-(3-(tert-

butyldimethylsilyloxy)propyl)pyrrolidine-1,2dicarboxylate

(2S,4R)-2-Benzyl 1-tert-butyl 4-(3-hydroxypropyl) pyrrolidine-1,2-dicarboxylate

(2S,4R)-2-Benzyl 1-tert-butyl 4-(3-methoxypropyl) pyrrolidine-1,2-dicarboxylate

( $\left.2 S, 3 S, 5 R, 6 S, 1^{\prime} R\right)$-3-Benzyloxy-2-benzyloxymethyl-

6-(1'-tert-butyldiphenylsilyloxyethyl)-1-

azabicyclo[3.2.0]heptan-7-one

$\left(2 S, 3 S, 5 R, 6 S, 1^{\prime} R\right)-3$-Benzyloxy-6-(1'-tert-

butyldimethylsilyloxyethyl)-2-hydroxymethyl-1-

azabicyclo[3.2.0] heptan-7-one

$\left(2 S, 3 S, 5 R, 6 S, 1^{\prime} R\right)-3$-Benzyloxy-6-(1'-tert-

butyldimethylsilyloxyethyl)-2-(p-methoxybenzyloxymethyl)-1-azabicyclo[3.2.0]heptan-7-one $\left(2 S, 3 S, 5 R, 6 S, 1^{\prime} R\right)-3$-Benzyloxy-6-(1'-tert-

781 butyldiphenylsilyloxyethyl)-2-hydroxymethyl-1azabicyclo[3.2.0]heptan-7-one

(2S,3S)-3-Benzyloxy-2-(p-methoxybenzyloxymethyl)- 781 3,4-dihydro-2H-pyrrole-1-oxide

Bisoxazolomycin A 1142

2-Bromo-4-((4-oxo-2-thioxo-1,3-thiazolidin-5- 1057 ylidene)methyl)phenyl 4-methoxybenzenesulfonate (2S,4R)-1-N-(tert-Butoxycarbonyl)-4-(3-(tert-butyldi- 888 methylsilyloxy)propyl)pyrrolidine-2-carboxylic acid

(2S,4R)-1-N-(tert-Butoxycarbonyl)-4-ibutylpyrrolidine-2-carboxylic acid

(2S,4R)-1-N-(tert-Butoxycarbonyl)-4(3-methoxypropyl)pyrrolidine-2-carboxylic acid (2S,4R)-1- $N$-(tert-Butoxycarbonyl)-4-((E)-pent-2enyl)pyrrolidine-2-carboxylic acid

(2S,4R)-1-N-(tert-Butoxycarbonyl)-4-npentylpyrrolidine-2-carboxylic acid

(2S,4R)-1- $N$-(tert-Butoxycarbonyl)-4-npropylpyrrolidine-2-carboxylic acid

2(S)-1- $N$-(tert-Butoxycarbonyl)-4,4-di- $n$ propylpyrrolidine-2-carboxylic acid

tert-Butyl(3-((2R,3R)-3-((E)-4,8-dimethylnona-3,7dien-1-yl)-2-methyloxiran-2-yl)propoxy) dimethylsilane tert-Butyl(3-((2R,3S)-3-(iodomethyl)-2-methyloxiran2-yl)propoxy)dimethylsilane tert-Butyldimethyl(3-((2R,3R)-2-methyl-3(4-methylpent-3-en-1-yl)oxiran-2-yl)propoxy)silane tert-Butyldimethyl(3-((2R,3R)-2-methyl-3-

$((3 E, 7 E, 11 E)-4,8,12,16$-tetramethylheptadeca3,7,11,15-tetraen-1-yl)oxiran-2-yl)propoxy)silane tert-Butyldimethyl(3-((2R,3R)-2-methyl-3-((3E,7E)4,8,12-trimethyltrideca-3,7,11-trien-1-yl)oxiran2-yl)propoxy)silane

((2R,3R)-3-(3-((tert-Butyldimethylsilyl)oxy)propyl)-3- 647 methyloxiran-2-yl)methanol

$\left(2 S, 3 S, 5 R, 6 S, 1^{\prime} R\right)-6-\left(1^{\prime}\right.$-tert-Butyldiphenylsilyl 781 oxyethyl)-3-hydroxy-2-hydroxymethyl-1-azabicyclo [3.2.0]heptan-7-one 
(2S,3S,5R,6S,1'R)-6-(1'-tert-Butyldiphenylsilyl oxyethyl)-3-hydroxy-2-trityloxymethyl-1-

azabicyclo[3.2.0] heptan-7-one

(2S,5R,6S,1'R)-6-(1'-tert-Butyldiphenylsilyloxyethyl)- 781

2-hydroxymethyl-1-azabicyclo[3.2.0] heptane-3,

7-dione

(2S,3S,5R,6S,1'R)-6-(1'-tert-Butyldiphenylsilyl

oxyethyl)-3-methanesulfonyloxy-

2-hydroxymethyl-1-azabicyclo[3.2.0]heptan-7-one

(2S,3S,5R,6S,1'R)-6-(1'-tert-Butyldiphenylsilyl

oxyethyl)-3-methanesulfonyloxy-

2-trityloxymethyl-1-azabicyclo[3.2.0]heptan-7-one

(5R,6S, 1'R)-6-(1'-tert-Butyldiphenylsilyloxyethyl)-

7-oxo-1-azabicyclo[3.2.0]hept-2-ene-2-

carbaldehyde

Calpinactam

CD101

Ceftriaxone

5-(4-Chlorophenyl)-2,8-dithioxo-2,3,7,8,9,

10-hexahydropyrido[2,3-d:6,5-d']dipyrimidine-4, $6(1 H, 5 H)$-dione

Chrysomycin A, 4'-acetyl-

Chrysomycin B, 4'-acetyl-

Citreorosein, 2'-acetoxy-7-chloro

Cladomarine

Clarithromycin

Clostomicin A, B , B $_{2}, \mathrm{C}, \mathrm{D}$

Cosmochlorin D, E

Cycloserine, D-

Cyperine

Diapolic acid A, B

8,13-Dihydroxy-3,7(11)-eudesmadien-12,8-olide

(S)-2-(5-(2,3-Dihydroxy-3-methylbutyl)-1 $H$-indol-

3-yl)acetonitrile

$\left(5 Z, 8 Z, 11 R^{*}, 12 R^{*}\right)-11,12-$ Dihydroxytetradeca-5, 991

8-dienamide

$(R, E)$-5,9-Dimethyl-1-((S)-2-methyltetrahydrofuran-

2-yl)deca-4,8-dien-1-ol

(E)-((3,7-Dimethylocta-2,6-dien-1-yl)sulfonyl)benzene 647

DNAC-2

EA-2801

Elmenol C, D, E, F, G, H

1053

601

Emodin, 1-O-methyl-6- $O$-( $\alpha$-D-ribofuranosyl)-

737

Erythromycin

527

Ethyl $(2 R, 3 S, 3 \mathrm{a} R, 5 S, 6 S)$-5-benzyloxy-6-

781

benzyloxymethyl-2-methylhexahydro-pyrrolo[1,2-b]

isoxazole-3-carboxylate

Ethyl $\left(2^{\prime} S, 3^{\prime} R\right)-2^{\prime}-(2 R, 4 S, 5 S)-(4-b e n z y l o x y-5-$

benzyloxymethyl-pyrrolidin-2-yl)-3'-hydroxy-

butanoate
Ethyl $\left(2^{\prime} S, 3^{\prime} R\right)-2^{\prime}-(2 R, 4 S, 5 S)-(4-$ benzyloxy-5-

781

benzyloxymethyl-pyrrolidin-2-yl)-3'-tert-

utyldiphenylsilyloxy-butanoate

Ethyl (2R,3S,3aR,5S,6S)-5-(benzyloxy)-6-

781

( $p$-methoxybenzyloxymethyl)-2-methylhexahydro-

pyrrolo[1,2-b]isoxazole-3-carboxylate

Ethyl $\left(2 R, 4 S, 5 S, 2^{\prime} S, 3^{\prime} R\right)$-2'-[4-benzyloxy-5-

781

( $p$-methoxybenzyloxymethyl)-pyrrolidin-2-yl]-3'-

tert-butyldimethylsilyloxy-butanoate

Ethyl (2R,4S,5S,2'S,3'R)-2'-[4-benzyloxy-5-

781

(p-methoxybenzyloxymethyl)-pyrrolidin-2-yl]-3'-

hydroxy-butanoate

F-36316 A, B 981

Fidaxomicin $\quad 492$

FKI-6817A $\quad 395$

Fradiamine A 611

Fusarielin J, K, L $\quad 726$

Ganoleucin D $\quad 915$

Germicidin H, I, J 200

GEX1A analogues $\quad 675$

Ghanamycin A, B 733

Grincamycin G, H 819

Herbicidin F, 8'-epi- 313

Hisunic acid 216

13-Hydroxy-3,5,8,7(11)-eudesmatetraen-12,8-olide 1029

13-Hydroxy-3,8,7(11)-eudesmatrien-12,8-olide 1029

2-Hydroxy-3-methyl-8-(methylamino)naphthalene- $\quad 320$

5,6-dione

5-(2-Hydroxynaphthalen-1-yl)2,8-dithioxo-2,3,7,8, 845

9,10-hexahydropyrido[2,3-d:6,5-d']dipyrimidine-4, $6(1 H, 5 H)$-dione

Hymerhabdrin A

1043

IFQ A, B, C, D, E, F, G

IIQ A, B, C 414

Indolecarbazole $\quad 715$

Integric acid, 13,13-dimethoxy 1129

Ionylideneacetic acid, (+)- $\gamma-$

Isariketide A acetate $\quad 856$

Isariketide B 856

Ivermectin $\quad 495$

Ivermectin $\mathrm{B}_{1 \mathrm{a}}, \mathrm{B}_{1 \mathrm{~b}} \quad 680$

Kifunensine $\quad 506$

Kribelloside A, B, C, D 582

781 Lariatin A 685

Lentzeoside A, B, C, D, E, F 448

Leptosphaerin H, I 743 
Lincomycin, 7(S)-7-acetylthio-1'- $N$-(tertbutoxycarbonyl)-1'-demethyl-7-deoxy

Lincomycin, 7(S)-1'-N-acetyl-1'-demethyl-7-deoxy- $\quad 888$ 7-[5-\{5-(methylamino)thiazol-4-yl $\}-1,3,4$-thiadiazol2-ylthio]

Lincomycin, 7(R)-7-acetylthio-7-deoxy

Lincomycin, 7(S)-7-(3'-amino-[1,1'-biphenyl]-4ylthio)-7-deoxy

Lincomycin, (7S)-7-[5-(5-amino-1-methyl- $1 \mathrm{H}$ pyrazol-4-yl)-1,3,4-thiadiazol-2-ylthio]-7-deoxy

Lincomycin, 7(S)-7-(5-amino-1,3,4-thiadiazol2-ylthio)-7-deoxy-1'-demethyl

Lincomycin, 7(S)-7-(6-aminobenzothiazol-2-ylthio)7-deoxy-1'-demethyl

Lincomycin, (7S)-7-[5-(2-aminophenyl)-1,3, 4-thiadiazol-2-ylthio]-7-deoxy

Lincomycin, (7S)-7-[5-(3-aminophenyl)-1,3, 4-thiadiazol-2-ylthio]-7-deoxy

Lincomycin, (7S)-7-[5-(4-aminophenyl)-1,3, 4-thiadiazol-2-ylthio]-7-deoxy

Lincomycin, 7(S)-4'-(3-aminopropyl)-1'-N-(tertbutoxycarbonyl)-1'-demethyl-7-deoxy-4'-depropyl7-\{5-(2-nitrophenyl)-1,3,4-thiadiazol-2-ylthio $\}$

Lincomycin, (7S)-7-[5-(3-aminopyrazin-2-yl)-1, 3,4-thiadiazol-2-ylthio]-7-deoxy

Lincomycin, 7(S)-7-(4-(6-aminopyridin-3-yl) phenylthio)-7-deoxy

Lincomycin, (7S)-7-[5-(2-aminopyridin-3-yl)-1,3, 4-thiadiazol-2-ylthio]-7-deoxy

Lincomycin, 7(S)-7-([1,1'-biphenyl]-4-ylthio)-7-deoxy

Lincomycin, 7(S)-7-(4-bromophenylthio)-7-deoxy

Lincomycin, $1^{\prime}-N$-(tert-butoxycarbonyl)-4'-\{3-(tertbutyldimethylsilyloxy)propyl $\}-1$ '-demethyl-4'depropyl

Lincomycin, 7(S)-1'-N-(tert-butoxycarbonyl)-7-\{4(carboxyl)phenylthio\}-1'-demethyl-7-deoxy

Lincomycin, 7(S)-1'-N-(tert-butoxycarbonyl)-7-\{4(carboxyl)phenylthio $\}-1^{\prime}$-demethyl-7-deoxy-4'depropyl-4'-n-pentyl

Lincomycin, $1^{\prime}-N$-(tert-butoxycarbonyl)-1'-demethyl

Lincomycin, 7(S)-1'-N-(tert-butoxycarbonyl)-1'demethyl-7-deoxy-4'-depropyl-4'-

(3-hydroxypropyl)-7-\{5-(2-nitrophenyl)-1, 3,4-thiadiazol-2-ylthio

Lincomycin, 7(S)-1'-N-(tert-butoxycarbonyl)-1'demethyl-7-deoxy-4'-depropyl-7-(4(methoxycarbonyl)phenyl)thio-4'-\{(E)-pent-2-enyl $\}$

Lincomycin, 7(S)-1'-N-(tert-butoxycarbonyl)-1'demethyl-7-deoxy-4'-depropyl-7-\{4(methoxycarbonyl)phenylthio \}-4'-n-pentyl

Lincomycin, 7(S)-1'-N-(tert-butoxycarbonyl)-1'demethyl-7-deoxy-4'-depropyl-7-\{4(morpholinocarbonyl)phenylthio \}-4'-n-pentyl

888

888

655

655

655

888

655

655
Lincomycin, 7(S)-1'-N-(tert-butoxycarbonyl)-1'demethyl-7-deoxy-7-mercapto

Lincomycin, 7(S)-1'-N-(tert-butoxycarbonyl)-1'demethyl-7-deoxy-7-\{4-(methoxycarbonyl) phenylthio

Lincomycin, 7(S)-1'-N-(tert-butoxycarbonyl)-1'-

888 demethyl-7-deoxy-7-[5-\{5-(methylamino)thiazol4-yl\}-1,3,4-thiadiazol-2-ylthio]-2,3,4-tris- $O$ (trimethylsilyl)

Lincomycin, 7(S)-1'-N-(tert-butoxycarbonyl)-1'demethyl-7-deoxy-7-\{4-(morpholinocarbonyl) phenylthio

Lincomycin, 7(S)-1'-N-(tert-butoxycarbonyl)-1'demethyl-7-deoxy-7-\{(4-(pyridin-3-yl)phenylthio $\}$

Lincomycin, 7(S)-1'-N-(tert-butoxycarbonyl)-1'demethyl-7-deoxy-7-\{4-(pyrimidin-5-yl)phenylthio $\}$

Lincomycin, $1^{\prime}-N$-(tert-butoxycarbonyl)-1'demethyl-4'-depropyl-4'- $i$-butyl

Lincomycin, $1^{\prime}-N$-(tert-butoxycarbonyl)-1'demethyl-4'-depropyl-4'-i-butyl-2,3,4-tris- $O$ (trimethylsilyl)

Lincomycin, 1'- $N$-(tert-butoxycarbonyl)-1'demethyl-4'-depropyl-4'-\{03-(tertbutyldimethylsilyloxy)propyl $\}$-2,3,4-tris- $O$ (trimethylsilyl)

Lincomycin, 1'- $N$-(tert-butoxycarbonyl)-1'demethyl-4'-depropyl-4'-(3-methoxypropyl)

Lincomycin, $1^{\prime}-N$-(tert-butoxycarbonyl)-1'demethyl-4'-depropyl-4'-(3-methoxypropyl)-2, 3,4-tris- $O$-(trimethylsilyl)

Lincomycin, $1^{\prime}-N$-(tert-butoxycarbonyl)-1'demethyl-4'-depropyl-4'-\{(E)-pent-2-enyl $\}$

Lincomycin, $1^{\prime}-N$-(tert-butoxycarbonyl)-1'demethyl-4'-depropyl-4'-\{(E)-pent-2-enyl $\}-2$, 3,4-tris- $O$-(trimethylsilyl)

Lincomycin, $1^{\prime}-N$-(tert-butoxycarbonyl)-1'demethyl-4'-depropyl-4"- $n$-pentyl

Lincomycin, $1^{\prime}-N$-(tert-butoxycarbonyl)-1'demethyl-4'-depropyl-4'-n-pentyl-2,3,4-tris- $O$ (trimethylsilyl)

Lincomycin, 1'- $N$-(tert-butoxycarbonyl)-1'demethyl-4'-propyl

Lincomycin, 1'- $N$-(tert-butoxycarbonyl)-1'demethyl-4'-propyl-2,3,4-tris- $O$-(trimethylsilyl) demethyl-2,3,4-tris- $O$-(trimethylsilyl)

Lincomycin, 7(S)-4'-i-butyl-1'-demethyl-7deoxy-4'-depropyl-7-(5-(2-nitrophenyl)-1, 3,4-thiadiazol-2-yl)thio

Lincomycin, 7(S)-1'- $N$-\{2-(tert-butyldimethylsilyloxy) 888 ethyl $\}$-1'-demethyl-7-deoxy-7-[5-\{5-(methylamino) thiazol-4-yl\}-1,3,4-thiadiazol-2-ylthio] 
Lincomycin, 7(S)-1'-N-\{2-(tert-butyldimethylsilyloxy) 888 ethyl $\}-1$ '-demethyl-7-deoxy-7-\{5-(2-nitrophenyl)-1, 3,4-thiadiazol-2-ylthio

Lincomycin, (7S)-7-[5-(2-carbamoylphenyl)-1,3, 4-thiadiazol-2-ylthio]-7-deoxy

Lincomycin, 7(S)-7-chloro-7-deoxy-2,3,4-tris- $O$ (trimethylsilyl)

Lincomycin, (7S)-7-[5-(2-chlorophenyl)-1,3, 4-thiadiazol-2-ylthio]-7-deoxy

Lincomycin, 7(S)-7-(3'-cyano-[1,1'-biphenyl]4-ylthio)-7-deoxy

Lincomycin, (7S)-7-[5-(2-cyanophenyl)-1,3, 4-thiadiazol-2-ylthio]-7-deoxy

Lincomycin, 7(S)-7-(4-(5-cyanopyridin-3-yl) phenylthio)-7-deoxy

Lincomycin, 7(S)-1'-demethyl-7-deoxy-4'-depropyl- 888 $4^{\prime}$-(3-dimethylaminopropyl)-7-\{5-(2-nitrophenyl)-1, 3,4-thiadiazol-2-ylthio

Lincomycin, 7(S)-1'-demethyl-7-deoxy-4'-depropyl- 888 4'-(3-methoxypropyl)-7-\{5-(2-nitrophenyl)-1,3, 4-thiadiazol-2-ylthio

Lincomycin, 7(S)-1'-demethyl-7-deoxy-4'-depropyl- 888 7-[5-\{5-(methylamino)thiazol-4-yl $\}-1,3,4-$ thiadiazol-2-ylthio]-4'-n-pentyl

Lincomycin, 7(S)-1'-demethyl-7-deoxy-4'-depropyl7-\{4-(morpholinocarbonyl)phenylthio $\}-4^{\prime}-n$ pentyl

Lincomycin, 7(S)-1'-demethyl-7-deoxy-4'-depropyl7-\{5-(2-nitrophenyl)-1,3,4-thiadiazol-2-ylthio $\}-4^{\prime}$ $n$-pentyl

Lincomycin, 7(S)-1'-demethyl-7-deoxy-1'-N-(2hydroxyethyl)-7-[5- $\{5-($ methylamino)thiazol-4-yl $\}$ -1,3,4-thiadiazol-2-ylthio]

Lincomycin, 7(S)-1'-demethyl-7-deoxy-1'-N-(2hydroxyethyl)-7-\{5-(2-nitrophenyl)-1,3,4thiadiazol-2-ylthio $\}$

Lincomycin, 7(S)-1'-demethyl-7-deoxy-1'- $N-\{2(R)-$ hydroxypropyl $\}-7-[5-\{5-($ methylamino)thiazol-4-yl $\}$ 1,3,4-thiadiazol-2-ylthio]

Lincomycin, 7(S)-1'-demethyl-7-deoxy-7-[5-\{5(methylamino)thiazol-4-yl $\}-1,3,4-$ thiadiazol2-ylthio]

Lincomycin, 7(S)-1'-demethyl-7-deoxy-7-[5-\{5(methylamino)thiazol-4-yl \}-1,3,4-thiadiazol-2ylthio]-1'-N-(4-methylthiazol-5-ylmethyl)

Lincomycin, 7(S)-1'-demethyl-7-deoxy-7-\{4(morpholinocarbonyl)phenylthio

Lincomycin, 7(S)-1'-demethyl-7-deoxy-7-\{5(2-nitrophenyl)-1,3,4-thiadiazol-2-yl thio- $1^{\prime}-N-i$ propyl

Lincomycin, 7(S)-1'-demethyl-7-deoxy-7-\{5(2-nitrophenyl)-1,3,4-thiadiazol-2-ylthio \}
655

52

655

655

888

888

Lincomycin, 7(S)-1'-demethyl-7-deoxy-4'-n-

888 propyl-7-\{5-(2-nitrophenyl)-1,3,4-thiadiazol2-ylthio $\}$

Lincomycin, 7(S)-1'-demethyl-7-deoxy-7-\{(4(pyridin-3-yl)phenylthio

Lincomycin, 7(S)-1'-demethyl-7-deoxy-7-\{4(pyrimidin-5-yl)phenylthio

Lincomycin, 7(S)-7-deoxy-4'-depropyl-7-[5-\{5(methylamino)thiazol-4-yl\}-1,3,4-thiadiazol-2ylthio]-4'-n-pentyl

Lincomycin, 7(S)-7-deoxy-4'-depropyl-7-\{4(morpholinocarbonyl)phenylthio $\}-4^{\prime}-n$-pentyl

Lincomycin, 7(S)-7-deoxy-4'-depropyl-7-\{5(2-nitrophenyl)-1,3,4-thiadiazol-2-ylthio \}-4'-npentyl

Lincomycin, 7(S)-7-deoxy-7-(4-(2-(dimethylamino) ethyl)phenylthio)

Lincomycin, 7(S)-7-deoxy-7-(4-((dimethylamino) methyl)phenylthio)

Lincomycin, 7(S)-7-deoxy-7-(4-(3-(dimethylamino) prop-1-yn-1-yl)phenylthio)

Lincomycin, 7(S)-7-deoxy-7-(4-(3-(dimethylamino) propyl)phenylthio)

Lincomycin, (7S)-7-deoxy-7-[5-(2dimethylcarbamoylphenyl)-1,3,4-thiadiazol-2-ylthio]

Lincomycin, 7(S)-7-deoxy-7-(4-(5-fluoropyridin-3-yl) phenylthio)

Lincomycin, (7S)-7-deoxy-7-[5-(2-furanyl)-1,3, 4-thiadiazol-2-ylthio]

Lincomycin, 7(S)-7-deoxy-7-(4-(1H-imidazol-1-yl) phenylthio)

Lincomycin, 7(R)-7-deoxy-7-mercapto

888

888

888

888

888

Lincomycin, 7(S)-7-deoxy-7-(3'-methoxy-[1, 1'-biphenyl]-4-ylthio)

Lincomycin, (7S)-7-deoxy-7-\{5-[2(methoxycarbonyl)phenyl]-1,3,4-thiadiazol-2-ylthio $\}$

Lincomycin, 7(S)-7-deoxy-7-(4-((2(S)-(methoxymethyl) 52 pyrrolidin-1-yl)methyl)phenylthio)

Lincomycin, (7S)-7-deoxy-7-[5-(2-methoxyphenyl) -1,3,4-thiadiazol-2-ylthio]

Lincomycin, 7(S)-7-deoxy-7-(4-(2-methoxypyridin -3-yl)phenylthio)

Lincomycin, 7(S)-7-deoxy-7-(4-(5-methoxypyridin -3-yl)phenylthio)

Lincomycin, 7(S)-7-deoxy-7-(4-(6-methoxypyridin -3-yl)phenylthio)

Lincomycin, (7S)-7-deoxy-7-[5-(1-methyl-4-nitro -1H-pyrazol-3-yl)-1,3,4-thiadiazol-2-ylthio]

Lincomycin, (7S)-7-deoxy-7-[5-(1-methyl-5-nitro -1H-pyrazol-4-yl)-1,3,4-thiadiazol-2-ylthio]

Lincomycin, 7(S)-7-deoxy-7-(4-(1-methyl-1,2,5, 6-tetrahydropyridin-3-yl)phenylthio) 655

655 
Lincomycin, (7S)-7-deoxy-7-\{5-[2-(methylamino) phenyl]-1,3,4-thiadiazol-2-ylthio $\}$

Lincomycin, 7(S)-7-deoxy-7-[5-\{5-(methylamino) thiazol-4-yl $\}-1,3,4$-thiadiazol-2-ylthio]

Lincomycin, 7(S)-7-deoxy-7-(4-(2-(4methylpiperazin-1-yl)ethyl)phenylthio)

Lincomycin, 7(S)-7-deoxy-7-(4-((4-methylpiperazin -1-yl)methyl)phenylthio)

Lincomycin, 7(S)-7-deoxy-7-(4-(1-methylpiperidin -3-yl)phenylthio)

Lincomycin, (7S)-7-deoxy-7-\{5-[2-(methylsulfonyl) phenyl]-1,3,4-thiadiazol-2-ylthio $\}$

Lincomycin, (7S)-7-deoxy-7-\{5-[2-(methylthio) phenyl]-1,3,4-thiadiazol-2-ylthio $\}$

Lincomycin, (7S)-7-deoxy-7-[5-(2-naphtyl)-1,3, 4-thiadiazol-2-ylthio]

Lincomycin, (7S)-7-deoxy-7-[5-(2-nitrophenyl)-1, 3,4-thiadiazol-2-ylthio]

Lincomycin, (7S)-7-deoxy-7-[5-(3-nitrophenyl)-1, 3,4-thiadiazol-2-ylthio]

Lincomycin, (7S)-7-deoxy-7-[5-(4-nitrophenyl)-1, 3,4-thiadiazol-2-ylthio]

Lincomycin, 7(S)-7-deoxy-7-(4-(piperidin-1-ylmethyl) phenylthio)

Lincomycin, 7(S)-7-deoxy-7-(4-(piperidin-3-yl) phenylthio)

Lincomycin, 7(S)-7-deoxy-7-(4-(pyrazin-2-yl) phenylthio)

Lincomycin, 7(S)-7-deoxy-7-(4-(pyridin-2-yl) phenylthio)

Lincomycin, 7(S)-7-deoxy-7-(4-(pyridin-3-yl) phenylthio)

Lincomycin, 7(S)-7-deoxy-7-(4-(pyridin-4-yl) phenylthio)

Lincomycin, (7S)-7-deoxy-7-[5-(2-pyridyl)-1, 3,4-thiadiazol-2-ylthio]

Lincomycin, (7S)-7-deoxy-7-[5-(3-pyridyl)-1, 3,4-thiadiazol-2-ylthio]

Lincomycin, (7S)-7-deoxy-7-[5-(4-pyridyl)-1, 3,4-thiadiazol-2-ylthio]

Lincomycin, 7(S)-7-deoxy-7-(4-(pyrimidin-2-yl) phenylthio)

Lincomycin, 7(R)-7-deoxy-7-(4-(pyrimidin-5-yl) phenylthio)

Lincomycin, 7(S)-7-deoxy-7-(4-(2-(pyrrolidin-1-yl) ethyl)phenylthio)

Lincomycin, 7(S)-7-deoxy-7-(4-(pyrrolidin-1-ylmethyl) 52 phenylthio)

Lincomycin, 7(S)-7-deoxy-7-(4-(1,2,3-thiadiazol-4-yl) 52 phenylthio)

Lincomycin, 7(S)-7-deoxy-7-(4-(1,3,4-thiadiazol-2-yl) 52 phenylthio)

888
Lincomycin, 7(S)-7-deoxy-7-(4-(thiazol-4-yl) phenylthio)

Lincomycin, (7S)-7-deoxy-7-[5-(2-thienyl)-1,3, 4-thiadiazol-2-ylthio]

Lincomycin, (7S)-7-deoxy 7-[5-(o-tolyl)-1,3, 4-thiadiazol-2-ylthio]

Lincosaminide, methyl (7S)-7-deoxy-7-[5-(4,5dimethoxy-2-nitrophenyl)-1,3,4-thiadiazol-2yl-thio]-6- $N-\left[\left(2^{\prime} S, 4^{\prime} R\right)-4^{\prime}\right.$-(n-propyl)piperidine$2^{\prime}$-carbonyl]- $\alpha$-thio

Lincosaminide, methyl (7S)-7-deoxy-6- $N-\left[\left(2^{\prime} S\right.\right.$, $4^{\prime} R$ )-4-ethyl-1'- $N$-methylpiperidine-2'-carbonyl] -7-[5-(4-fluoro-2-nitrophenyl)-1,3,4-thiadiazol -2-ylthio]- $\alpha$-thio

Lincosaminide, methyl (7S)-7-deoxy-6- $N-\left[\left(2^{\prime} S\right.\right.$, $\left.4^{\prime} R\right)-4^{\prime}$-ethylpiperidine-2'-carbonyl]-7-[5-(4, 5-dimethoxy-2-nitrophenyl)-1,3,4-thiadiazol2-ylthio]- $\alpha$-thio

Lincosaminide, methyl (7S)-7-deoxy-6- $N-\left[\left(2^{\prime} S\right.\right.$, $4^{\prime} R$ )-4'-ethylpiperidine-2'-carbonyl]-7-[5-(4-fluoro2-nitrophenyl)-1,3,4-thiadiazol-2-yl-thio]- $\alpha$-thio

Lincosaminide, methyl (7S)-7-deoxy-6- $N-\left[\left(2^{\prime} S\right.\right.$, $\left.4^{\prime} R\right)-4^{\prime}$-ethylpiperidine-2'-carbonyl]-7-[5-(2nitrophenyl)-1,3,4-thiadiazol-2-yl-thio]- $\alpha$-thio

Lincosaminide, methyl (7S)-7-deoxy-7-[5-(4-fluoro2-nitrophenyl)-1,3,4-thiadiazol-2-yl-thio]-6- $N$ $\left[\left(2^{\prime} S, 4^{\prime} R\right)-1^{\prime}-N\right.$-methyl-4'-(n-propyl)piperidine$2^{\prime}$-carbonyl]- $\alpha$-thio

Lincosaminide, methyl (7S)-7-deoxy-7-[5-(4-fluoro2-nitrophenyl)-1,3,4-thiadiazol-2-yl-thio]-6- $N-\left[\left(2^{\prime} S\right.\right.$, $\left.4^{\prime} R\right)-1^{\prime}-N$-methyl-4'-(n-propyl)piperidine-2'carbonyl]- $\alpha$-thio

Lincosaminide, methyl (7S)-7-deoxy-7-[5-(4-fluoro2-nitrophenyl)-1,3,4-thiadiazol-2-yl-thio]-6- $N$ $\left[\left(2^{\prime} S, 4^{\prime} R\right)-4^{\prime}-(n\right.$-propyl)piperidine-2'-carbonyl]$\alpha$-thio

Lincosaminide, methyl (7S)-7-deoxy-7-[5-(5methoxy-2-nitrophenyl)-1,3,4-thiadiazol-2yl-thio]-6- $N-\left[\left(2^{\prime} S, 4^{\prime} R\right)-4^{\prime}\right.$-(n-propyl)piperidine2 '-carbonyl]- $\alpha$-thio

Lincosaminide, methyl (7S)-7-deoxy-7-[5-(5methylamino-2-nitrophenyl)-1,3,4-thiadiazol2-yl-thio]-6- $N$-[(2'S, $\left.4^{\prime} R\right)-4^{\prime}$-(n-propyl)piperidine2 '-carbonyl]- $\alpha$-thio

Lincosaminide, methyl (7S)-7-deoxy-7-[5-(2nitrophenyl)-1,3,4-thiadiazol-2-ylthio]-6- $N$ [(2'R, 4'S)-4'-(n-propyl)piperidine-2-carbonyl]$\alpha$-thio

Lincosaminide, methyl (7S)-7-deoxy-7-[5-(2nitrophenyl)-1,3,4-thiadiazol-2-ylthio]-6- $N$ [(2'S, 4'R)-4'-(n-propyl)piperidine-2'-carbonyl]$\alpha$-thio
52

655

655

1112

1112

1112

1112

1112 


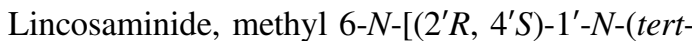
butoxycarbonyl)- $4^{\prime}-\left(n\right.$-butyl)piperidine- $2^{\prime}$ carbonyl]- $\alpha$-thio

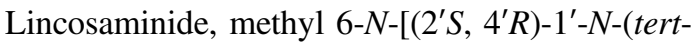
butoxycarbonyl)- $4^{\prime}-\left(n\right.$-butyl)piperidine- $2^{\prime}$ carbonyl]- $\alpha$-thio

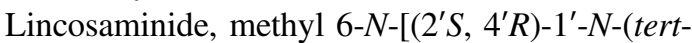
butoxycarbonyl)-4'-(n-butyl)piperidine-2'carbonyl]-2,3,4-tris- $O$-(trimethylsilyl)- $\alpha$-thio

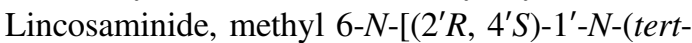
butoxycarbonyl)-4'-ethylpiperidine-2'-carbonyl]$\alpha$-thio

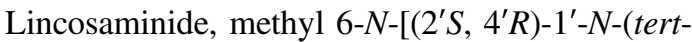
butoxycarbonyl)- $4^{\prime}$-ethylpiperidine- $2^{\prime}$-carbonyl]$\alpha$-thio

Lincosaminide, methyl $6-N-\left[\left(2^{\prime} S, 4^{\prime} R\right)-1^{\prime}-N-(\right.$ tert butoxycarbonyl)- $4^{\prime}$-ethylpiperidine- $2^{\prime}$-carbonyl]-2, 3,4-tris- $O$-(trimethylsilyl)- $\alpha$-thio

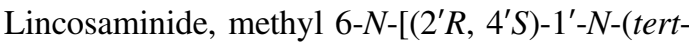
butoxycarbonyl)- $4^{\prime}-\left(n\right.$-propyl)piperidine- $2^{\prime}$ carbonyl]- $\alpha$-thio

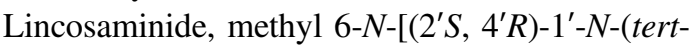
butoxycarbonyl)- $4^{\prime}-\left(n\right.$-propyl)piperidine- $2^{\prime}$ carbonyl]- $\alpha$-thio

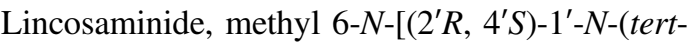
butoxycarbonyl)-4'-(n-propyl)piperidine- $2^{\prime}$ carbonyl]-2,3,4-tris- $O$-(trimethylsilyl)- $\alpha$-thio

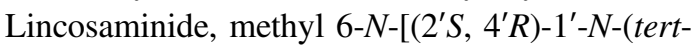
butoxycarbonyl)- $4^{\prime}$-(n-propyl)piperidine- $2^{\prime}$ carbonyl]-2,3,4-tris- $O$-(trimethylsilyl)- $\alpha$-thio

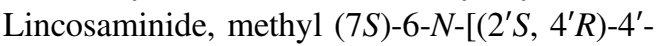
(n-butyl)-1'- $N$-methylpiperidine- $2^{\prime}$-carbonyl]-7deoxy-7-[5-(4-fluoro-2-nitrophenyl)-1,3, 4-thiadiazol-2-yl-thio]- $\alpha$-thio

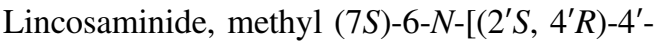
(n-butyl)piperidine-2'-carbonyl]-7-deoxy-7-[5(4,5-dimethoxy-2-nitrophenyl)-1,3,4-thiadiazol2-ylthio]- $\alpha$-thio

Lincosaminide, methyl (7S)-6- $N-\left[\left(2^{\prime} S, 4^{\prime} R\right)-4^{\prime}-\right.$ (n-butyl)piperidine-2'-carbonyl]-7-deoxy-7-[5(4-fluoro-2-nitrophenyl)-1,3,4-thiadiazol-2-yl-thio]$\alpha$-thio

Lincosaminide, methyl $(7 S)-6-N-\left[\left(2^{\prime} S, 4^{\prime} R\right)-4^{\prime}-\right.$ (n-butyl)piperidine-2'-carbonyl]-7-deoxy-7-[5-(2nitrophenyl)-1,3,4-thiadiazol-2-yl-thio]- $\alpha$-thio

Lincosaminide, methyl 7-deoxy-7-methyl-6- $N$ $\left[\left(2^{\prime} R, 4^{\prime} S\right)-4^{\prime}-(n\right.$-propyl)piperidine-2'-carbonyl]$\alpha$-thio

Lincosaminide, methyl 7-deoxy-7-methyl-6- $N-\left[\left(2^{\prime} S\right.\right.$, $\left.4^{\prime} R\right)-4^{\prime}$-(n-propyl)piperidine- $2^{\prime}$-carbonyl]- $\alpha$-thio

Lysocin E

MA026
1112

Maoxianamide A, B

187

MBJ-0173, -0174

226

1-Methoxy-3,5'-dimethyl-2,3'-oxybiphenyl-5, 1',2'-triol 331

1112

5-(4-Methoxyphenyl)-2,8-dithioxo-2,3,7,8,9,

845 10-hexahydropyrido [2,3-d:6,5-d']dipyrimidine-4,6 $(1 H, 5 H)$-dione

1112

5-Methoxy-3,5'-dimethyl-2,3'-oxybiphenyl-1,1',2'-triol 331

Methyl (2R,3S,5R,6S,1'R)-3-(benzoyloxy)-6-(1'-(tert- 781 butyldimethylsilyloxyethyl)-7-oxo-1-azabicyclo

1112 [3.2.0]heptane-2-carboxylate

Methyl $\left(5 R, 6 S, 1^{\prime} R\right)-6-\left(1^{\prime}\right.$-tert-

butyldiphenylsilyloxyethyl)-7-oxo-1-azabicyclo

1112 [3.2.0]hept-2-ene-2-carboxylate

Methyl (E)-3-((2-carbamimidoylhydrazono)methyl)1-(4-chlorobenzyl)-1H-indole-6-carboxylate

1112 hydrochloride

Methyl (E)-1-(4-chlorobenzyl)-3-((2-(4,5-dihydro$1 H$-imidazol-2-yl)hydrazono)methyl)- $1 H$-indole-

1112 5-carboxylate hydrobromide

Methyl (E)-3-((2-(4,5-dihydro-1H-imidazol-2-yl) hydrazono)methyl)-1-(4-fluorobenzyl)- $1 H$-indole-

1112 6-carboxylate hydrobromide

(R)-5-Methyl-1-((S)-2-methyltetrahydrofuran-2-yl) hex-4-en-1-ol

$1112(5 S, 6 S)-5-M e t h y l-6-((3 E, 7 E)-4,8,12$-trimethyltrideca3,7,11-trien-1-yl)-1-azabicyclo[3.1.0]hexane

$S$-(3-((2R,3R)-2-Methyl-3-((3E,7E)-4,8, 781

12-trimethyltrideca-3,7,11-trien-1-yl)oxiran-2-yl) propyl) ethanethioate

2-(3-((2R,3R)-2-Methyl-3-((3E,7E)-4,8,

1112 12-trimethyltrideca-3,7,11-trien-1-yl)oxiran-2-yl) propyl)isoindoline-1,3-dione

3-((2R,3R)-2-Methyl-3-((3E,7E)-4,8,12trimethyltrideca-3,7,11-trien-1-yl)oxiran-2-yl)

1112 propyl 4-methylbenzenesulfonate

(2R,3R)-3-Methyl-2-((3E,7E)-4,8,12-trimethyltrideca-

3,7,11-trien-1-yl)tetrahydro-2 $H$-thiopyran-3-ol

((3-Methylbut-2-en-1-yl)sulfonyl)benzene

6-Methylsalicylic acid

Milbemycin A3, 4-hydroxy- $\Delta^{2,3}$ -

Milbemycin A4, 4-hydroxy- $\Delta^{2,3}$ -

Monocyclofarnesol, (-)-trans- $\gamma$ -

MSD-819

Mycaminosyltylonolide, 20-deoxy-20- $\{N$-benzyl-

Mycaminosyltylonolide, 20-deoxy-20- $\{N$-methyl-

Nafithromycin

Naphthacemycin $A_{1}, A_{10}, A_{11}, A_{2}, A_{3}, A_{4}, A_{5}, \quad 562,568$ 691 $N$-[1-(3-quinolyl)-1H-1,2,3-triazol-4-yl] methylamino $-5-O$ -

$N$-[1-(3-quinolyl)-1H-1,2,3-triazol-4-yl]

methylamino $-5-O$ -

647

$A_{6}, A_{7}, A_{8}, A_{9}, B_{1}, B_{2}, B_{3}, B_{4}, C_{1}, C_{2}$ 
Naphthacemycin A9, ( \pm )-

NC1404

Neihumicin, isomethoxy

5-(4-Nitrophenyl)-2,8-dithioxo-2,3,7,8,9, 10-hexahydropyrido[2,3-d:6,5-d']dipyrimidine 4,6 (1H,5H)-dione

5-(4-Nitrophenyl)-2,8-dithioxo-2,3,7,8,9, 10-hexahydropyrido[2,3-d:6,5-d']dipyrimidine 4,6 $(1 H, 5 H)$-dione

Nocathiacin analogs

Nocazine F, G

Norvancomycin derivatives

Ogipeptin A, B, C, D

Oligomycin A, 33-dehydro

2-One-13-hydroxy-3,5,8,7(11)-eudesmatetraen-12, 8-olide

OP0595

Opantimycin A

Oxacillin

Oxazolomycin A, A2

Oxirapentyn L

Pacritaxel

Pargamicin B, C, D

Penicillenol A1, A2, B1, B2, C1, C2

Petasitol, 11-O-methyl

Phellidene E

Phenazine, 1-hydroxy-

Phenazine-1-carboxylic acid

Phenazine-1-carboxylic acid, 2-hydroxy-

Phenazine-1-carboxylic acid, 2-methoxy-

Phosalacine

Pikromycin

Prodiginine, propyl-meta-cyclooctyl

Prodigiosin, metacyclo

Propeptin

Propeptin-2

Pseudopyronine B

Pteridic acid C, D, E, F, G

5-(Pyridin-2-yl)-2,8-dithioxo-2,3,7,8,9, 10-hexahydropyrido[2,3-d:6,5-d']dipyrimidine-4,6 $(1 H, 5 H)$-dione

5-(Pyridin-2-yl)-2,8-dithioxo-2,3,7,8,9,

10-hexahydropyrido[2,3-d:6,5-d']dipyrimidine-4,6 $(1 H, 5 H)$-dione

Pyripyropene A

Quinoline-5,8-dione, 6-amino-2,3-dimethyl

Quinoline-5,8-dione, 2,3-dimethyl-6-(methylamino)

Quinoline-5,6-dione, 2,3-dimethyl-8-(methylamino)

Quinoline-6-one, 5-hydroxy-2,3-dimethyl-8-

(methylamino)-5-(2-oxopropyl)
574

970

590

845

845

671

795

158

7984

871

1029

246

222

771

1142

856

506

699

763

1133

695

1146

1146

1146

1146

506

527

196

109, 196

685

685

96

1047

845
Reblastatin, 7-O-descarbamoyl-17-O-demethyl

323

RK-144171

Roxithromycin

Rubrumol

Saccharothriolide G, H

Sacrolide A, 15,16-dihydro

Sacrolide A, 9-epi-

Secalonic acid F1

Setamycin

Simamycin

Solithromycin

Spirotoamide C, D

102

527

1138

Stemphol C, D

Sterenoid A, B, C, D, E, F, G, H, I, J, K, L

TAN-1120, 6"-cyano-6"-deoxy-

718

708

708

967

506

607

527

710

737

1104

Tegaserod

219

918

Teicoplanin pseudoaglycon derivatives

Telithromycin

152,664

Tenvermectin A, 4"-O-glucosyl

Tenvermectin B, 4"-O-glucosyl

Terfestatin D

527

190

190

987

Terpentecin $\quad 506$

Tetracenoquinocin A 1026

$(R, 4 E, 8 E, 12 E)-5,9,13,17-T e t r a m e t h y l-1-((S)-2-$ methyltetrahydrofuran-2-yl)octadeca-4,8,12, 16-tetraen-1-ol

(((2E,6E,10E)-3,7,11,15-Tetramethylhexadeca-2, 6,10,14-tetraen-1-yl)sulfonyl)benzene

Theaflavin

Theaflavin-3, 3'-O, $O$-digallate

Theaflavin-3-O-gallate

Thiazomycin

Thiazomycin analogs

647

Tiacumicin A, B, C, D, E, F

Tirandamycin A, B

TP0017383

TP0020827

TP0083177

TP0097302

647

443

443

443

671

671

492

798

264

264

264

Trachyspic acid 19-butyl ester

Trichocyclodipeptide A, B, C 923

Trichopeptide A, B 923

Trienylfuranol A $\quad 721$

Trienylfuranone A, B 721

3-(3,4,5-Trihydroxy-6-(hydroxymethyl)tetrahydro-2H- 754 pyran-2-yloxy)propane-1,2-diyl dipalmitate

$(R, 4 E, 8 E)-5,9,13-$ Trimethyl-1- $((S)-2-$

647

methylpyrrolidin-2-yl)tetradeca-4,8,12-trien-1-ol

$(R, 4 E, 8 E)-5,9,13$-Trimethyl-1-((S)-2-methyltetrahy- 647 drothiophen-2-yl)tetradeca-4,8,12-trien-1-ol

Tylosin

527 
Tyropeptin A, B

Tyropeptin-boronic acid derivatives

Tyrostatin

\section{Vancomycin}

Versicone E, F, G, H

Vestaine $\mathrm{A}_{1}, \mathrm{~B}_{2}$
542

542

506

174

179
Waldiomycin

251

Xanthen-9-one, 4-chloro-1-hydroxy-3-methoxy6-methyl-8-methoxycarbonyl-

Xantholipin B 